\title{
Rotating Fulde-Ferrell-Larkin-Ovchinnikov state in cold Fermi gases
}

\author{
Tomohiro Yoshida and Youichi Yanase \\ Department of Physics, Niigata University, Niigata 950-2181, Japan
}

(Dated: November 3, 2018)

\begin{abstract}
We study an effect of rotation on the Fulde-Ferrell-Larkin-Ovchinnikov (FFLO) state of two component Fermi superfluid gases in a toroidal trap. We investigate a stability of the FFLO states in the quasi-one-dimensional regime on the basis of the Bogoliubov-de Gennes equation. We find that two novel FFLO phases, i.e., the half quantum vortex state and the intermediate state of FuldeFerrell (FF) state and Larkin-Ovchinnikov (LO) state, are stabilized by the rotation. The phase diagram for the FF state, LO state, intermediate state, and half quantum vortex state is shown in both $T$ - $P$ plane and $T$ - $h$ plane. We demonstrate characteristic features of these states, such as the order parameter, flux quantization, and local polarization. Several related works are discussed, and the advantages of cold Fermi gases are indicated.

PACS numbers: 67.85.-d, 71.10.Ca, 74.25.Dw
\end{abstract}

\section{INTRODUCTION}

Two component Fermi gases with population imbalance attract much attention from both theoretical and experimental point of view [1 3$]$. One of the motivations of current studies is the realization of FuldeFerrell-Larkin-Ovchinnikov (FFLO) state [4, 5], which is characterized by a spatial oscillation of the order parameter arising from the center-of-mass momentum of Cooper pairs. This state is induced by a mismatch of the Fermi surfaces between two component Fermi particles produced by a population imbalance. Since many parameters, such as the population imbalance, interaction strength, and trap potential, can be experimentally controlled [3], cold Fermi gases are regarded as a promising candidate for the FFLO superfluid. Recent experiment found an evidence for the FFLO superfluid state in the elongated harmonic trap [6], and attract much attention in a variety of fields such as the condensed matter physics [7-15] and nuclear physics [16] where possible FFLO phases have been discussed.

The FFLO state has an internal degree of freedom arising from the inversion symmetry, and therefore, there are several phases of the FFLO state. Most of theoretical works on the FFLO state are focused on the two phases. One is the Fulde-Ferrell (FF) state which is described by the order parameter $\Delta(\mathbf{r}) \propto \mathrm{e}^{i \mathbf{q} \cdot \mathbf{r}}$, and the other is the Larkin-Ovchinnikov (LO) state in which $\Delta(\mathbf{r}) \propto \cos (\mathbf{q} \cdot \mathbf{r})$. The LO state is regarded as a mixture of two FF states with opposite momentum $\Delta(\mathbf{r}) \propto \mathrm{e}^{i \mathbf{q} \cdot \mathbf{r}}$ and $\Delta(\mathbf{r}) \propto \mathrm{e}^{-i \mathbf{q} \cdot \mathbf{r}}$. It has been shown that the LO state is stable against the FF state in most cases [9].

The FFLO state in cold Fermi gases has been studied in the literature [17 24]. Some of their works investigated the effect of harmonic trap potential which plays an essential role for the spatial structure of the FFLO phase. They found that the radial FFLO (R-FFLO) state is stable, in which the order parameter changes its sign along the radial direction around the edge of the harmonic trap. The spatial symmetry remaining in the harmonic trap, however, is not broken in the R-FFLO state, thus it may be difficult to experimentally observe the spatial oscillation of order parameter. On the other hand, one of us showed that the angular FFLO (A-FFLO) state in which the order parameter changes its sign along the angular direction is stable in a toroidal trap 25]. Since the A-FFLO state breaks the rotation symmetry, one expects that a spatial modulation characteristic of the FFLO state can be detected in experiments. Furthermore, A-FFLO state is a novel FFLO state in the sense that the rotation symmetry is spontaneously broken. This is in sharp contrast to the superconductors where the rotation symmetry is broken by the crystal lattice and therefore the A-FFLO state is not stabilized. Hence, it is interesting to study the response of A-FFLO state to the rotation. It is expected that another novel FFLO state is stabilized by the rotation. A related work has been given by Kashimura et al. They showed that a spontaneous current appears in a toroidal trap with a potential barrier [26], but they didn't investigate the effect of rotation. In this paper, we investigate the FFLO phases induced by the rotation. It is expected that those studies will be tested by the experiments since such controllable experiment is an advantage of cold atom gases.

An effect of rotation on the FFLO state has been studied in the context of cold Fermi gases $27-29]$ as well as in mesoscopic superconductors where the magnetic field plays an equivalent role to the rotation [28, 30, 31]. It has been shown that the nucleation of vortex in the FFLO superfluid gives rise to intriguing phenomena. In contrast to those works, our study is focused on the quasione-dimensional superfluid formed on the toroidal trap, which is an ideal system to study the effect of gauge field on the FFLO state. The A-FFLO state is regarded as the LO state along the quasi-one-dimensional ring, while order parameter of the vortex state is the same as that of the FF state. In this work, we study how the A-FFLO state formed on the toroidal trap is changed by the nucleation of vortex. The same issue has been investigated in the context of mesoscopic superconducting ring under the tilted magnetic field [32], but their study overlooked many important results, as we will show later. 
We show that the "giant vortex state" corresponding to the FF state is stabilized in the imbalanced gas near the superfluid critical temperature $T_{\mathrm{c}}$, and the phase transition to the "intermediate state" between the FF and LO states occurs with decreasing the temperature. Furthermore, the "half quantum vortex state" becomes stable in a certain range of the rotation and population imbalance. This phase leads to the half quantized flux of mass and the unusual Little-Parks oscillation of $T_{\mathrm{c}}$. We elucidate superfluid properties of these FFLO states, such as the superfluid order parameter, local polarization, and the flux of mass.

\section{FORMULATION}

We study rotating two component Fermi gases in a toroidal trap, in which atoms are loaded on a quasi-onedimensional ring [33, 34]. We assume that the angular velocity is perpendicular to the plane of ring, leading to $\boldsymbol{\Omega}=\Omega \hat{\mathbf{z}}$. Then, the Hamiltonian is approximately described by the following one-dimensional attractive Hubbard Hamiltonian:

$$
\begin{aligned}
H= & -t \sum_{j, \sigma}\left(\mathrm{e}^{i \Phi} \hat{c}_{j+1 \sigma}^{\dagger} \hat{c}_{j \sigma}+\mathrm{e}^{-i \Phi} \hat{c}_{j \sigma}^{\dagger} \hat{c}_{j+1 \sigma}\right) \\
& -|U| \sum_{j} \hat{n}_{j \uparrow} \hat{n}_{j \downarrow}-\sum_{j, \sigma} \mu_{\sigma} \hat{n}_{j \sigma},
\end{aligned}
$$

where $\sigma=\uparrow, \downarrow$ describe two hyperfine states, $\hat{n}_{j \sigma}=\hat{c}_{j \sigma}^{\dagger} \hat{c}_{j \sigma}$ is the number operator, $U$ is the coupling constant of attractive interaction, and $\mu_{\sigma}$ is the chemical potential for $\sigma$ particles. The mass of atoms $M$ is related to $t=$ $1 / 2 M$, and the phase $\Phi=R \Omega / 2 t$ denotes the Peierls phase, where $R$ is the radius of ring (see Appendix A). We take the unit $\hbar=k_{\mathrm{B}}=1$ and $t=1$.

Although one-dimensional models can be solved with use of sophisticated analytic or numerical methods $35-$ 42], we adopt the Bogoliubov-de Gennes (BdG) equation, because our interests are focused on the quasi-onedimensional regime where a three-dimensional long range order is achieved [43 45]. Then, the Hamiltonian is reduced to the mean field Hamiltonian

$$
\begin{aligned}
H_{\mathrm{m}}= & -t \sum_{j, \sigma}\left(\mathrm{e}^{i \Phi} \hat{c}_{j+1 \sigma}^{\dagger} \hat{c}_{j \sigma}+\mathrm{e}^{-i \Phi} \hat{c}_{j \sigma}^{\dagger} \hat{c}_{j+1 \sigma}\right)-\sum_{j, \sigma} \mu_{\sigma} \hat{n}_{j \sigma} \\
& +\sum_{j}\left(\Delta_{j} \hat{c}_{j \uparrow}^{\dagger} \hat{c}_{j \downarrow}^{\dagger}+\Delta_{j}^{*} \hat{c}_{j \downarrow} \hat{c}_{j \uparrow}\right)+\sum_{j} \frac{\left|\Delta_{j}\right|^{2}}{|U|}
\end{aligned}
$$

where $\Delta_{j} \equiv-|U|\left\langle\hat{c}_{j \downarrow} \hat{c}_{j \uparrow}\right\rangle$ is the order parameter. We focus on the weak coupling BCS region, and then the BdG equation is quantitatively appropriate. The Hartree term can be neglected there. We don't touch the BCS-BEC crossover region where higher order corrections beyond the mean field theory play important roles [3, 46, 47], although it is expected that the FFLO state is robust there [25, 48].
The mean field Hamiltonian is diagonalized with use of the following Bogoliubov transformation:

$$
\hat{c}_{j \uparrow}=\sum_{\nu} u_{j \uparrow}^{\nu} \hat{\gamma}_{\nu}, \hat{c}_{j \downarrow}=\sum_{\nu} u_{j \downarrow}^{\nu *} \hat{\gamma}_{\nu}^{\dagger},
$$

where $\hat{\gamma}_{\nu}^{\dagger}$ and $\hat{\gamma}_{\nu}$ are the creation and annihilation operators of quasiparticles, respectively. The wave function of quasiparticles $\left(u_{j \uparrow}^{\nu}, u_{j \downarrow}^{\nu}\right)$ satisfies the BdG equation

$$
\sum_{l}\left[\begin{array}{cc}
H_{j l \uparrow} & \Delta_{j} \delta_{j l} \\
\Delta_{j}^{*} \delta_{j l} & -H_{j l \downarrow}^{*}
\end{array}\right]\left[\begin{array}{l}
u_{l \uparrow}^{\nu} \\
u_{l \downarrow}^{\nu}
\end{array}\right]=E_{\nu}\left[\begin{array}{c}
u_{j \uparrow}^{\nu} \\
u_{j \downarrow}^{\nu}
\end{array}\right],
$$

where $H_{j l \sigma}=-t \mathrm{e}^{i \Phi} \delta_{j-1, l}-t \mathrm{e}^{-i \Phi} \delta_{j+1, l}-\mu_{\sigma} \delta_{j l}$.

The self-consistent equation for the order parameter is obtained as,

$$
\Delta_{j}=-|U| \sum_{\nu} u_{j \uparrow}^{\nu} u_{j \downarrow}^{\nu *} f\left(E_{\nu}\right),
$$

where $f\left(E_{\nu}\right)$ is the Fermi distribution function. We can refer to the analytic solution of BdG equation in one dimension [49, 50], however, we numerically solve the selfconsistent equation (Eqs. (4) and (5)) to investigate the thermodynamic stability of superfluid phases. Several self-consistent solutions corresponding to the metastable states are obtained. We determine the stable phase by comparing the free energy of those states. The free energy is evaluated to be

$$
\begin{aligned}
F= & E-T S \\
= & \sum_{\nu} E_{\nu} f\left(E_{\nu}\right)+\sum_{j}\left[\frac{\left|\Delta_{j}\right|^{2}}{|U|}-\mu_{\downarrow}\right] \\
& +T \sum_{\nu}\left\{f\left(-E_{\nu}\right) \log \left[f\left(-E_{\nu}\right)\right]+f\left(E_{\nu}\right) \log \left[f\left(E_{\nu}\right)\right]\right\} .
\end{aligned}
$$

We solve BdG equations for a fixed chemical potential $\mu_{\sigma}$ in the ground canonical ensemble and calculate the particle number and population imbalance which are expressed as $N=N_{\uparrow}+N_{\downarrow}$ and $P=\left(N_{\uparrow}-N_{\downarrow}\right) / N$, respectively. We obtain the number of $\sigma$ particles $N_{\uparrow}$ and $N_{\downarrow}$ as

$$
\begin{aligned}
& N_{\uparrow}=\sum_{j} n_{j \uparrow}=\sum_{j} \sum_{\nu}\left|u_{j \uparrow}^{\nu}\right|^{2} f\left(E_{\nu}\right), \\
& N_{\downarrow}=\sum_{j} n_{j \downarrow}=\sum_{j} \sum_{\nu}\left|u_{j \downarrow}^{\nu}\right|^{2}\left[1-f\left(E_{\nu}\right)\right],
\end{aligned}
$$

respectively. We define the "magnetic field" $h=\left(\mu_{\uparrow}-\right.$ $\left.\mu_{\downarrow}\right) / 2$ as the difference of chemical potential between $\uparrow$ and $\downarrow$ particles.

\section{NUMERICAL RESULTS}

We set $|U| / t=1.5$ and assume the chemical potential $\left(\mu=\left(\mu_{\uparrow}+\mu_{\downarrow}\right) / 2=-0.8\right)$ leading to $n_{j \sigma} \equiv\left\langle\hat{n}_{j \sigma}\right\rangle \lesssim 0.4$ so that the lattice Hamiltonian appropriately describes 
the continuum gas. The following results are obtained for the number of lattice sites $N_{\mathrm{L}}=200$ with imposing the periodic boundary condition. Note that the periodic boundary condition should be satisfied for a gas on the ring.

In all our results the order parameter $\Delta_{j}$ is approximately described as

$$
\Delta_{j}=\Delta_{+} \mathrm{e}^{i q_{+} j}+\Delta_{-} \mathrm{e}^{-i q_{-} j},
$$

where the center-of-mass momentum of Cooper pairs is $q_{ \pm}=2 \pi m_{ \pm} / N_{\mathrm{L}}$ with $m_{ \pm}$being an integer so as to satisfy the periodic boundary condition. The FF state is described by a set of order parameter $\left(\Delta_{+}, \Delta_{-}\right) \propto(1,0)$ or $\left(\Delta_{+}, \Delta_{-}\right) \propto(0,1)$, while $\left(\Delta_{+}, \Delta_{-}\right) \propto(1, \pm 1)$ with $m_{+}=m_{-}=m$ in the LO state.

\section{A. Rotating FFLO phases}

Before studying the effect of rotation, we discuss the phase diagram of gases at rest. In Fig. 1, we show the phase diagram for $\Phi=0$ in the $T-h$ plane as well as in the $T-P$ plane. We denote the superfluid critical temperature $T_{\mathrm{c}}\left(h, \Phi / \Phi_{0}\right)$ as a function of the magnetic field $h$ and the normalized phase factor $\Phi / \Phi_{0}$, where $\Phi_{0} \equiv 2 \pi / N_{\mathrm{L}}$. The temperature in Fig. 1 is normalized by the critical temperature of the balanced gas at rest, $T_{\mathrm{c}}(0,0)=0.0816$. Figure 1(a) shows that the BCS state is stable for $h<0.0915$ while the LO state is stabilized for $h>0.0915$ at $T=0$. Note that the order parameter of the LO state is described as $\Delta_{j} \propto \cos \left[\left(\Phi_{0} m\right) j\right]$. Since we consider the Fermi gas trapped on the ring, this LO state corresponds to the A-FFLO state studied in Ref. 25]. The integer $m$ increases with increasing the magnetic field $h[9]$. Although the FFLO state is stable for a huge magnetic field $h \gg T_{\mathrm{c}}(0,0)$ in our calculation, our study focuses on the moderate magnetic field $h \sim T_{\mathrm{c}}$ where $m \ll N_{\mathrm{L}}$. Figure 1 (b) shows that the BCS state is unstable in the imbalanced gas $P>0$ at $T=0$. This is because the BCS state does not have an excess particle owing to the excitation gap. We also find forbidden regimes (FR) in the $T-P$ plane since the particle number is finite and not fixed in our calculation based on the grand canonical ensemble. Another inhomogeneous superfluid state may be stabilized in the canonical ensemble, but that is beyond the scope of our paper.

We here turn to the rotating systems. Figure 2(a) shows the phase diagram in the $T-\Phi$ plane for a field $h=0.13$ where the LO state with $m=4$ is stable at rest, while Fig. 2(b) shows the phase diagram for $h=0$. While a conventional vortex state and usual Little Parks oscillation of $T_{\mathrm{c}}$ appear in the rotating BCS state (Fig. 2(b)), we see several intriguing features in the rotating FFLO state (Fig. 2(a)). First, the FF state with $\Delta_{+}=0$ or $\Delta_{-}=0$ is stabilized near the critical temperature in the rotating gas with $\Phi / \Phi_{0} \neq 0$. The two FF states $\Delta_{j} \propto \mathrm{e}^{i q j}$ and $\Delta_{j} \propto \mathrm{e}^{-i q j}$ are degenerate at rest, and therefore, the mixture of them, i.e., the LO state $\Delta_{j} \propto\left(\mathrm{e}^{i q j}+\mathrm{e}^{-i q j}\right)$ is stabilized at $T=T_{\mathrm{c}}$. On the other hand, the degeneracy is lifted by the rotation, and then one of the FF states is stabilized near $T_{\mathrm{c}}$. This FF state is regarded as a "giant vortex state" because the vorticity $m$ is much larger than the conventional one. The critical temperature is increased by the rotation since one of the FF states is stabilized. The FF state may be furthermore stabilized by the Hartree term, which is neglected here, because of the Fermi liquid correction [51].

Second, the "half quantum vortex state" is stabilized by the rotation. Figure 2(a) shows that the LO state with $\left(m_{+}, m_{-}\right)=(4,4)$ changes to the phase $\left(m_{+}, m_{-}\right)=$ $(5,4)$, as the rotation, i.e., the phase $\Phi$ increases. The latter phase is regarded as the half quantum vortex state since the order parameter is approximately described at low temperatures as

$$
\begin{aligned}
\Delta_{j} & \propto \exp \left[i\left(\Phi_{0} \times 5\right) j\right]+\exp \left[-i\left(\Phi_{0} \times 4\right) j\right] \\
& \propto \exp \left[i\left(\Phi_{0} \times 0.5\right) j\right] \cos \left[\left(\Phi_{0} \times 4.5\right) j\right] .
\end{aligned}
$$

The exponential provides the phase $\pi$ for a circuit along the ring, which corresponds to a half quantized vortex. Another phase $\pi$ required for the uniqueness of order parameter is given by the odd number of phase changes in the sinusoidal function. Then, the order parameter of the half quantum vortex state is regarded as the product of those in the FF and LO states with an unconventional period. Similarly, the phase with $\left(m_{+}, m_{-}\right)=(6,3)$ is the half quantum vortex state while the phases with $\left(m_{+}, m_{-}\right)=(5,3)$ and $\left(m_{+}, m_{-}\right)=(6,2)$ are the LO state having one and two quantized vortex, respectively. Thus, the half quantum vortex state is realized when the difference of vorticity is odd between the coexisting $\mathrm{FF}$ states. One of the two quantum numbers $m_{+}$and $m_{-}$ changes with the increase of rotation, and induces the half quantized vortex. This is in sharp contrast to the rotating BCS state which has only one quantum number $m$. In other words, the half quantized vortex is induced by the multi-component order parameters of the FFLO state, as in the chiral $p$-wave superconductor [52].

We see a characteristic feature of the half quantum vortex state appearing in the quantized flux of mass. We show the rotation dependence of the flux of mass $f$ for $h=0$ and $h=0.13$ at $T=0$ in Fig. 3, which is obtained as

$$
\begin{aligned}
f & =f_{\uparrow}+f_{\downarrow}, \\
f_{\uparrow} & =2 t \Im\left[\sum_{\nu} u_{j \uparrow}^{\nu *} u_{j+1 \uparrow}^{\nu} f\left(E_{\nu}\right)\right], \\
f_{\downarrow} & =2 t \Im\left\{\sum_{\nu} u_{j \downarrow}^{\nu} u_{j+1 \downarrow}^{\nu *}\left[1-f\left(E_{\nu}\right)\right]\right\} .
\end{aligned}
$$

Since the flux is independent of the site, we omit the index $j$ in Eqs. (11) and (12). Figure 3 clearly shows that the flux of mass is half quantized in the rotating FFLO state $(h=0.13)$.

Owing to the nucleation of half quantum vortex, the $T_{\mathrm{c}}$ shows an unconventional Little-Parks oscillation studied 
(a)

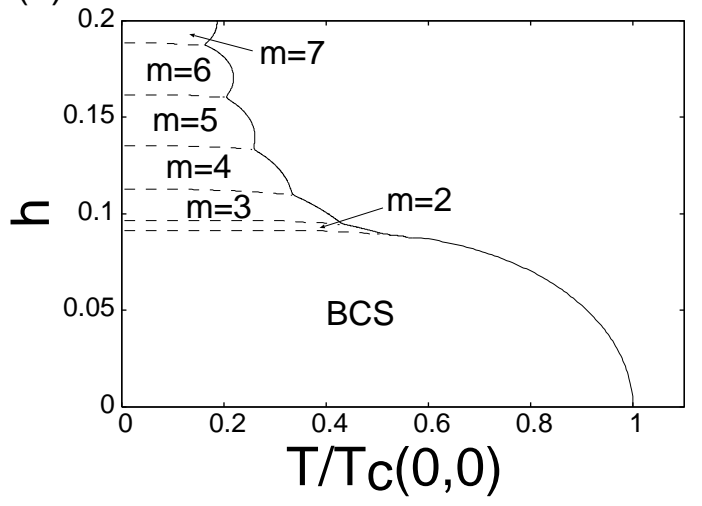

(b)

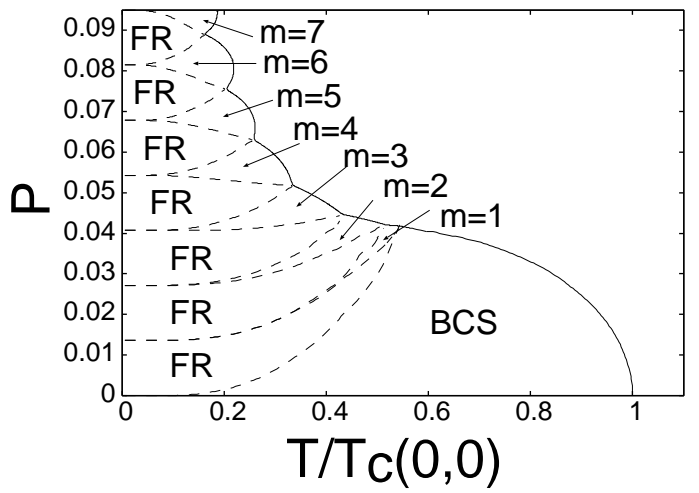

FIG. 1. (a) $T-h$ and (b) $T-P$ phase diagram for the gas at rest $\left(\Phi / \Phi_{0}=0\right)$. The integer $m$ denotes a center-of-mass momentum of Cooper pairs $q=\Phi_{0} \mathrm{~m}$. The solid and dashed lines show the second and first order transition lines, respectively. The BCS superfluid state with $m=0$ is denoted as "BCS", while $m>0$ for the LO state is shown in the figure. The character "FR" denotes the forbidden regimes in Fig. 1(b). The LO state with $m=1$ is stabilized in a tiny region of the $T-h$ plane, but it cannot be resolved in Fig. 1(a).

(a)

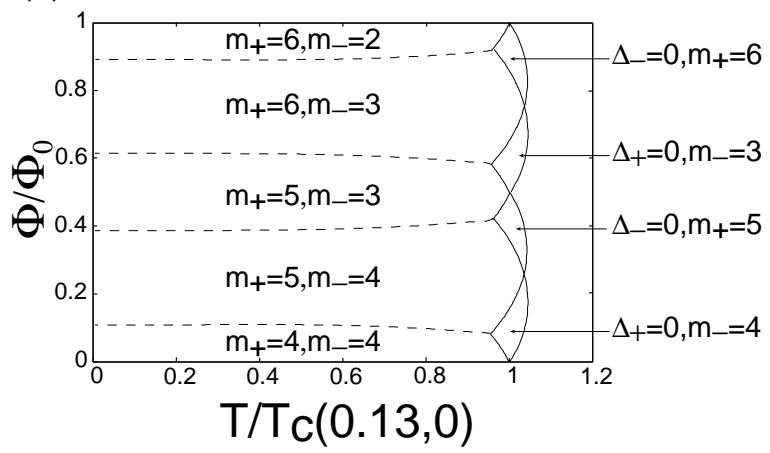

(b)

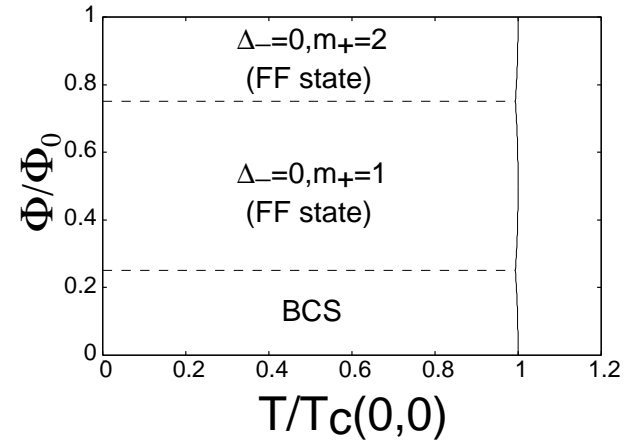

FIG. 2. $T-\Phi$ phase diagram at (a) $h=0.13$ and (b) $h=0$. The solid and dashed lines show the second and first order transition lines, respectively. The integers $m_{ \pm}$denote the momentum of Cooper pairs, and $\Delta_{ \pm}$are the amplitude of order parameter for $m_{ \pm}$, respectively (see Eq. (8)). The phase diagram is periodic for $\Phi$ with the period $\Phi_{0}$ (see Appendix B).

in Ref. 31]. Figure 2(a) shows the Little Parks oscillation with the period being a half of the conventional one. It should be noticed that the amplitude of oscillation is remarkably enhanced in the FFLO state compared with the BCS state.

Third, we point out another phase in Fig. 2(a), that is the mixture of LO state and FF state. For instance, the FFLO state with $\left(m_{+}, m_{-}\right)=(4,4)$ is not a pure LO state in the sense that the amplitude $\Delta_{+}$for $m_{+}=4$ is not equivalent to $\Delta_{-}$for $m_{-}=4$. The temperature dependence of the ratio $\delta \equiv \Delta_{+} / \Delta_{-}$at $h=0.13$ and $\Phi / \Phi_{0}=0.0637$ is shown in Fig. 4. where the superfluid phase is divided into three regions, (I), (II), and (III). In the low temperature region (I), the ratio $\delta$ is almost unity corresponding to the LO state, while the FF state with $\delta=0$ is stabilized in the high temperature region (III). Interestingly, the intermediate ratio $0<\delta<1$ is obtained in a wide intermediate temperature region (II). Thus, the "intermediate state" between the FF and LO states is stabilized by the rotation, and then the order parameter is described as

$$
\Delta_{j} \propto \delta \exp \left[i\left(\Phi_{0} \times 4\right) j\right]+\exp \left[-i\left(\Phi_{0} \times 4\right) j\right] .
$$

Although the intermediate state changes to the LO state through the crossover, these states can be distinguished by observing the flux of mass. As shown in Fig. 4, the flux of mass is almost zero in the LO state. This is because the susceptibility to the rotation is suppressed by the excitation gap. A narrow band is formed by the Andreev bound states localized around the nodes of order parameter [45], its contribution is negligible for these parameters. On the other hand, the flux of mass increases with temperature in the region (II), and shows a peak at the transition temperature to the FF state, $T=T_{\mathrm{c} 2}=0.946 T_{\mathrm{c}}(0.13,0.0637)$. The flux decreases as approaching to the superfluid critical temperature $T=T_{\mathrm{c}}$ because the order parameter of $\mathrm{FF}$ state is decreased. In other words, the flux of mass is first enhanced below $T_{\mathrm{c}}$ owing to the formation of the giant vortex state, and then suppressed below $T_{\mathrm{c} 2}$ as decreas- 


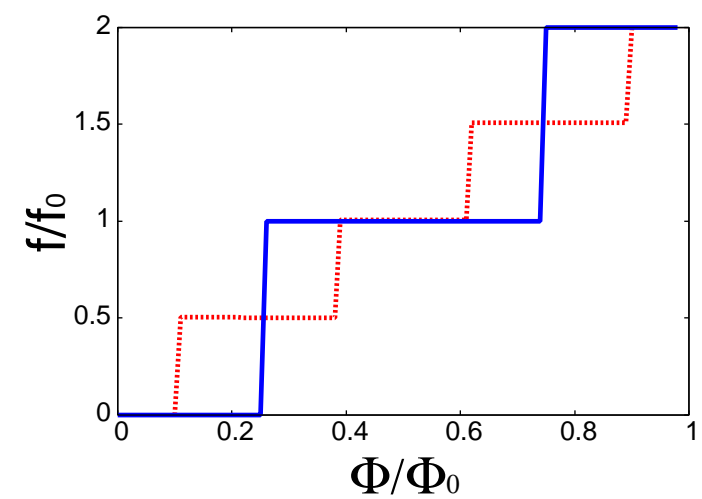

FIG. 3. (Color online) The rotation dependence of the flux of mass at $T=0$ in the BCS state ( $h=0$, blue solid line) and in the FFLO state ( $h=0.13$, red dotted line). The flux of mass is normalized by the value at $h=0$ and $\Phi / \Phi_{0}=0.5$.

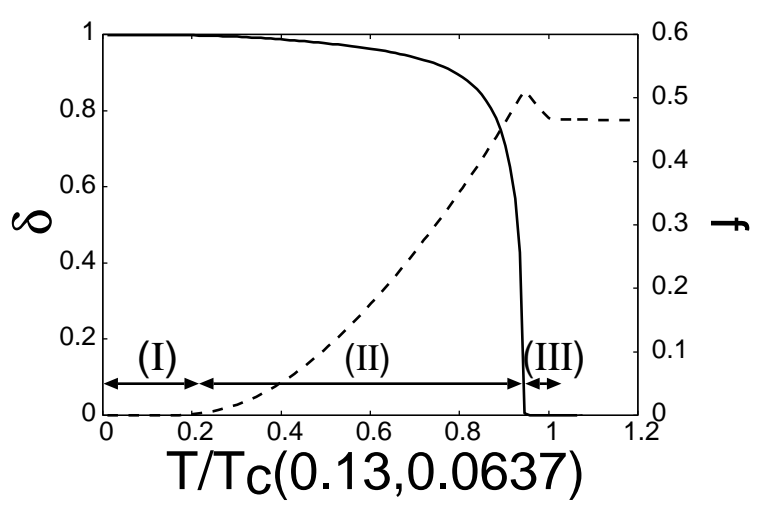

FIG. 4. Temperature dependence of the ratio $\delta \equiv \Delta_{+} / \Delta_{-}$ (solid line) and the flux of mass $f$ (dashed line) at $h=0.13$ and $\Phi / \Phi_{0}=0.0637$.

ing the temperature. Such nonmonotonic temperature dependence is a characteristic feature of the FFLO state, while that is not observed in the BCS superfluid. Note that we here assume the system without conserving the angular momentum. Another phase diagram will be obtained when the angular momentum is conserved across the superfluid transition.

\section{B. Local polarization}

The FFLO phases studied in the previous subsection show their characteristic feature in the local polarization. Figure 5 shows the spatial profile of local polarization $p_{j}=n_{j \uparrow}-n_{j \downarrow}$ in the $\mathrm{FF}, \mathrm{LO}$, half quantum vortex, and intermediate states. It is shown that the local polarization in the LO state shows peaks at the quasinodal points of order parameter where $\left|\Delta_{j}\right| \sim 0$ (compare Fig. (5)with Fig. [6), as in the A-FFLO state [25]. This is because excess particles are localized around the quasinodal points of order parameter in order to make the loss of condensation energy as small as possible. There- fore the number of peaks in the local polarization $n_{0}$ can be regarded as the number of quasi-nodal points in the order parameter. On the other hand, the FF state shows a uniform polarization, since the translation symmetry is not broken there. The spatial inhomogeneity is smoothly enhanced by decreasing the temperature in the intermediate state. Thus, we can discriminate the LO state, intermediate state, and FF state by the polarization measurements.

A characteristic feature of the half quantum vortex state appears in the number of peaks in the local polarization. The number $n_{0}$ is odd (even) in the half (integer) quantum vortex state. (See above discussion and Fig. 6.) For example, Fig. [5 (a) shows $n_{0}=8$, while that is $n_{0}=9$ in Fig. F(b) where the amplitude is described by $\left|\Delta_{j}\right| \propto\left|\cos \left[\left(\Phi_{0} \times 4.5\right) j\right]\right|$. Thus, the odd number of $n_{0}$ is a clear signature of the half quantum vortex state. Note that the $n_{0}$ is the same as the number of excess particles. As increasing the excess particles, the number of quasi-nodes increases so as to hold them.

\section{Mesoscopic effect}

We here comment on the magnetic field dependence of superfluid phases. Although the LO state with $\left(m_{+}, m_{-}\right)=(4,4)$ is stabilized at rest in both magnetic fields $h=0.115$ and $h=0.13$, the FF state induced by the rotation depends on the field. While the FF state with $\Delta_{+}=0$ is stabilized for $h=0.13$ (Fig. 2(a)), the other FF state with $\Delta_{-}=0$ is stabilized for $h=0.115$ as shown in Fig. 7. Accompanied by the change of FF state, the half quantum vortex state also changes as increasing the magnetic field from $h=0.115$ to $h=0.13$; a small rotation stabilizes the half quantum vortex state $\left(m_{+}, m_{-}\right)=(4,3)$ in the former, while $\left(m_{+}, m_{-}\right)=(5,4)$ in the latter.

We understand these magnetic field dependences in the following two ways. First, the superfluid phase having a large number of zeros of order parameter $n_{0}$ is stabilized by increasing the magnetic field so as to gain the magnetic energy. For example, $n_{0}=7\left[\left(m_{+}, m_{-}\right)=(4,3)\right]$ at $h=0.115$ increases to $n_{0}=9\left[\left(m_{+}, m_{-}\right)=(5,4)\right]$ at $h=0.13$.

Another interpretation is based on the discrete single particle spectrum, that is the mesoscopic effect. Figure 8 shows the schematic sketch of the Cooper pairing for the cases $h=0.115$ and $h=0.13$. The upper panel shows the Fermi momentum at rest, while the lower panel shows the shift of Fermi momentum due to the rotation (see Appendix A). This shift gives rise to the lifting of degeneracy of two FF states with $\Delta_{+}=0$ and $\Delta_{-}=0$. In Fig. 8 (a), a small shift moves the Cooper pairs with $m_{-}=4$ away from the Fermi surface. Since the Cooper pairs should be formed by the quasiparticles near the Fermi surface, the FF state with $m_{+}=4$ is favored at low magnetic fields as shown in Fig. 8 (a), while the other FF state with $m_{-}=4$ is stabilized at high magnetic fields as shown in 
(a)

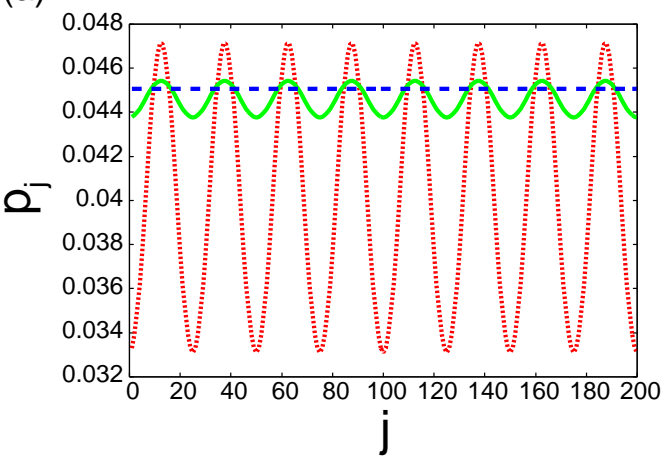

(b)

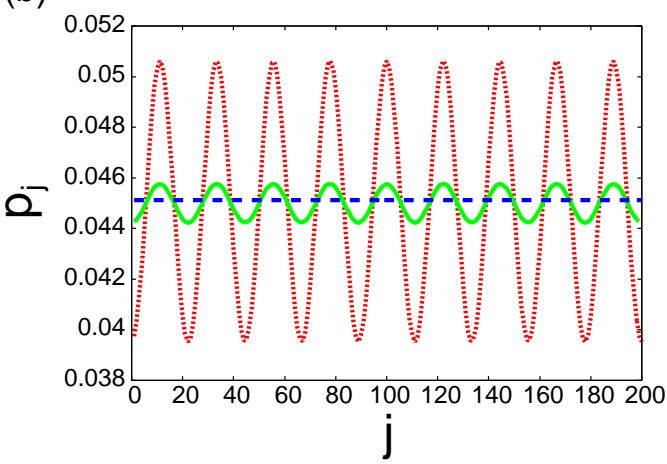

FIG. 5. (Color online) Spatial profile of the local polarization at $h=0.13$. (a) $\Phi / \Phi_{0}=0.0637$. The dotted (red), solid (green), and dashed (blue) lines represent the data for $T / T_{\mathrm{c}}(0.13,0)=0$ (LO state), $T / T_{\mathrm{c}}(0.13,0)=0.947$ (intermediate state), and $T / T_{\mathrm{c}}(0.13,0)=0.991$ (FF state), respectively. (b) $\Phi / \Phi_{0}=0.159$. The dotted (red), solid (green), and dashed (blue) lines represent the data for $T / T_{\mathrm{c}}(0.13,0)=0$ (half quantum vortex state), $T / T_{\mathrm{c}}(0.13,0)=0.969$ (intermediate state), and $T / T_{\mathrm{c}}(0.13,0)=1.01$ (FF state), respectively.

(a)

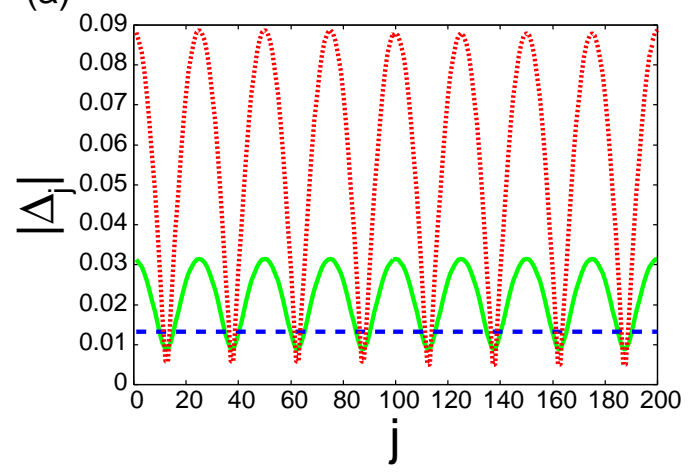

(b)

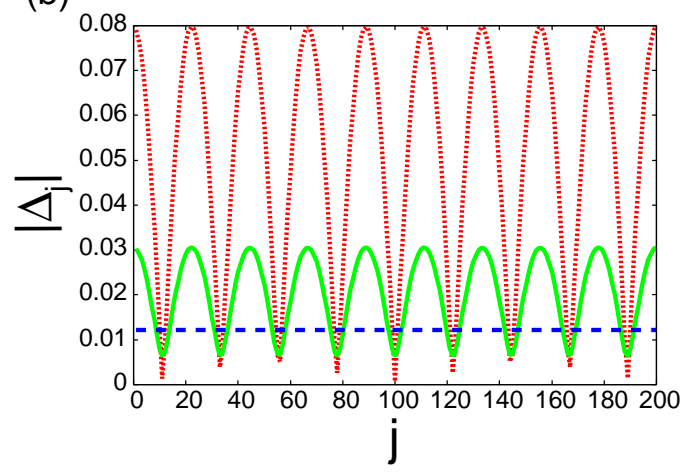

FIG. 6. (Color online) Spatial profile of the amplitude of order parameter $\left|\Delta_{j}\right|$ at $h=0.13$. In (a) and (b), the parameters are the same as in Figs. 5 (a) and (b), respectively.

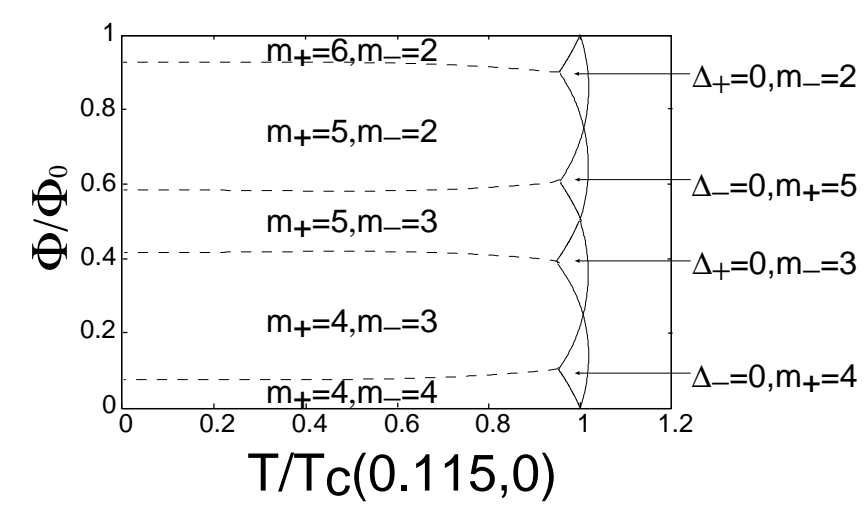

FIG. 7. $T-\Phi$ phase diagram at $h=0.115$. The other parameters are the same as Fig. 2

Fig. 8(b). In other words, the reconstruction of quasiparticle energy due to the rotation determines the stable FF state near $T_{\mathrm{c}}$. When the temperature is decreased, the sub-dominant order parameter appears below $T_{\mathrm{c} 2}$. That is $\Delta_{+}$with $m_{+}=4\left(\Delta_{-}\right.$with $\left.m_{-}=4\right)$ for a small rota- tion, and changes to $m_{+}=5\left(m_{-}=3\right)$ as increasing the rotation. Thus, the FF state with $\Delta_{+}=0$ changes to the half quantized vortex state $\left(m_{+}, m_{-}\right)=(5,4)$, while the other FF state with $\Delta_{-}=0$ changes to another half quantum vortex state $\left(m_{+}, m_{-}\right)=(4,3)$. Note again that these differences of the $T-\Phi$ phase diagram between $h=0.115$ and $h=0.13$ are induced by the mesoscopic effect.

\section{Phase diagram}

Finally, we discuss the phase diagram of the rotating Fermi superfluid gases in the $T-h$ and $T-P$ plane. We fix the rotation $\Phi / \Phi_{0}=0.286$ in Fig. 9. For this parameter the BCS state is changed to the vortex state with vorticity $m=1$. More important finding is that the half quantum vortex state [for example, $\left(m_{+}, m_{-}\right)=(1,0)$ ] is induced in a certain range of the population imbalance. Therefore, the flux of mass iteratively changes as $1 \rightarrow \frac{1}{2} \rightarrow 1 \rightarrow \frac{1}{2}$ with increasing the population imbalance. Another point is that the FF state is stabilized near 
(a)

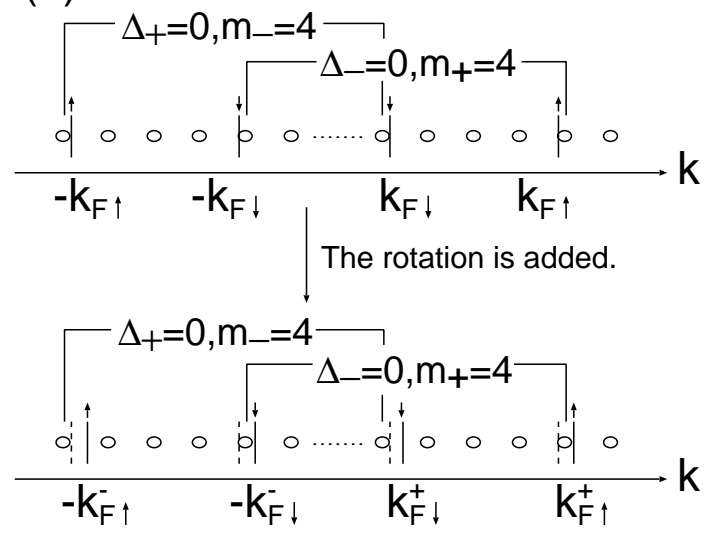

(b)
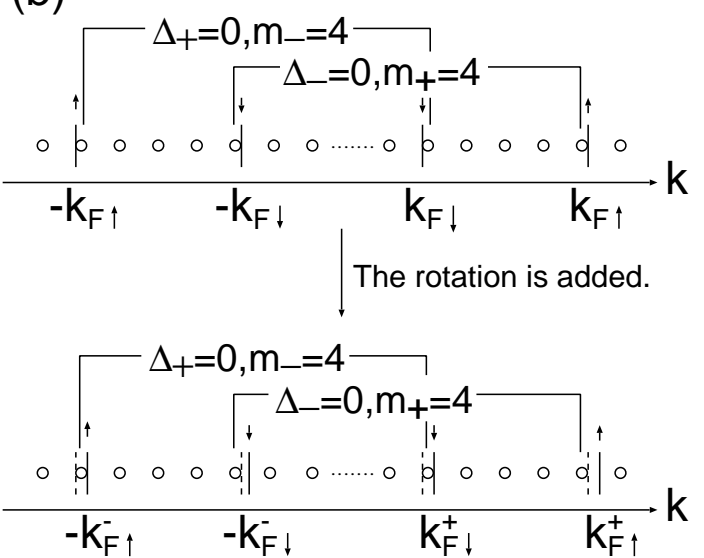

FIG. 8. Schematic sketch of Cooper pairing in the FFLO state. (a) the case of $h=0.115$ and (b) the case of $h=0.13$. The upper panel shows the quasiparticle energy of the gases at rest, while the lower panel shows the shift of Fermi momentum due to the rotation. The circles represent the momentum discretized by the mesoscopic effect. $k_{\mathrm{F} \sigma}$ and $k_{\mathrm{F} \sigma}^{ \pm}$are the Fermi momentum for $\sigma$ particles.

(a)

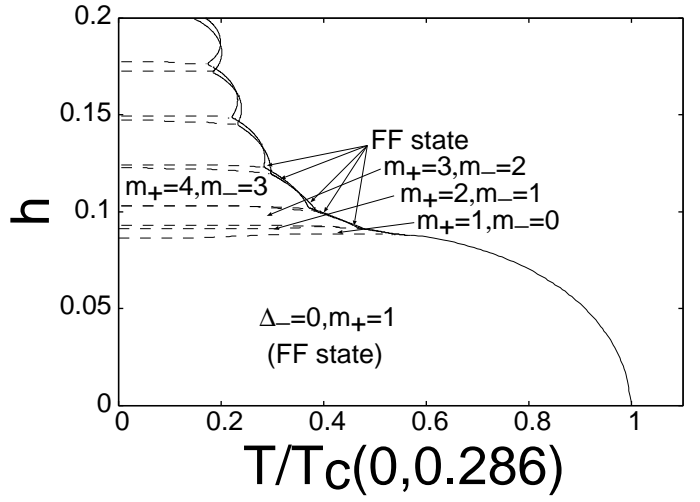

(c)

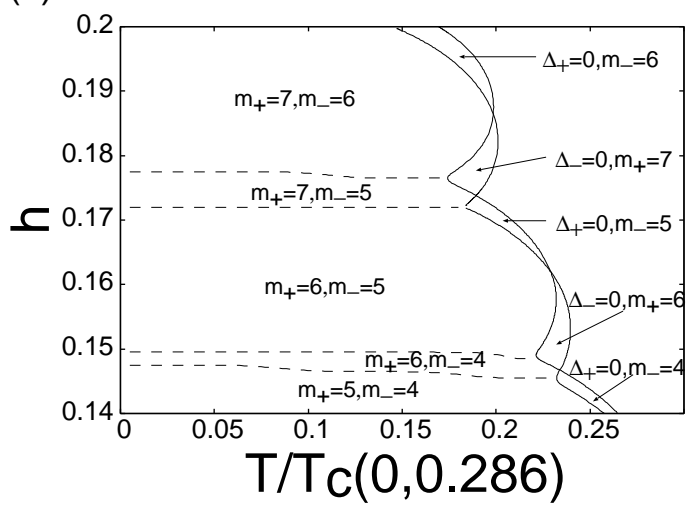

(b)

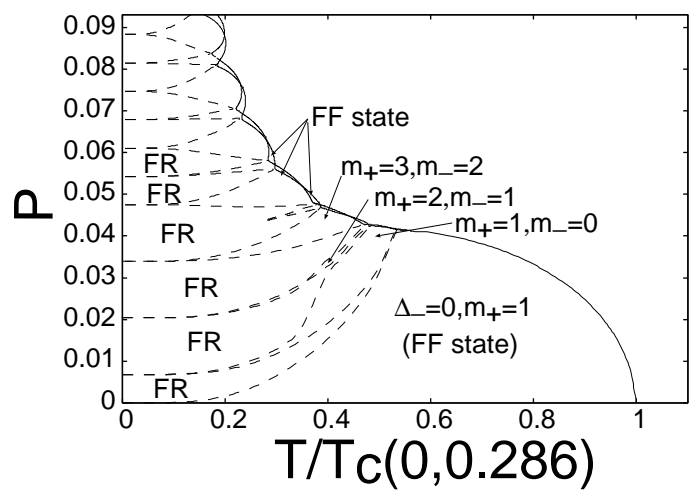

(d)

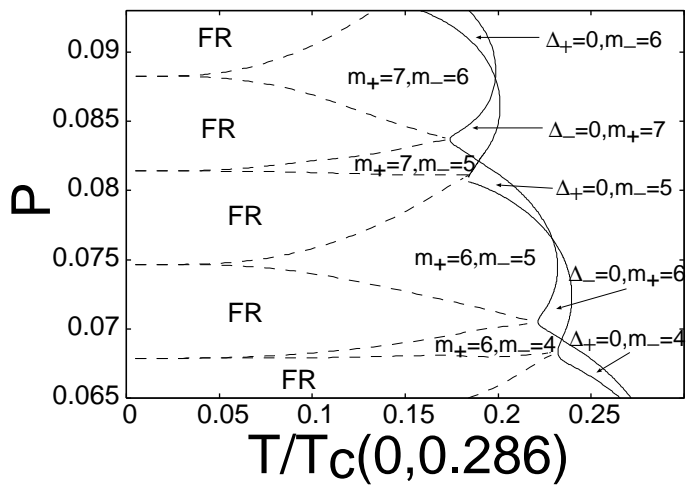

FIG. 9. (a) $T-h$ and (b) $T-P$ phase diagram for $\Phi / \Phi_{0}=0.286$. Scaled-up figures of (a) and (b) are shown in (c) and (d), respectively.

the critical temperature, as studied in section IIIA In order to illuminate these features, we show the phase diagram scaled-up in the large population imbalance (magnetic field) region. We see that the population imbalance changes the vorticity on a sequence of phase transition lines. These features are understood on the basis of the mesoscopic effect discussed in section [IIC.

For a rotation smaller than $\Phi / \Phi_{0}=0.286$, where the vortex is not nucleated in the balanced gas, a half quantum vortex can be induced by the population imbalance 
(compare Fig. 2(a) with Fig. 2(b)). Then, the gas begins to rotate by increasing the population imbalance, if the angular momentum is not conserved for some reasons. This intriguing phenomenon would be a signature of the FFLO superfluid gas.

\section{SUMMARY AND DISCUSSION}

We have studied effects of rotation on the FFLO superfluid state of imbalanced Fermi gases loaded on a quasione-dimensional toroidal trap. We found several novel phase transitions induced by the rotation and population imbalance. First, the FF state is stabilized in the rotating gases near the critical temperature, although this state is unstable against the LO state in the gases at rest. This means that the "giant vortex" appears in the imbalanced gases near $T_{\mathrm{c}}$ because the $\mathrm{FF}$ state on the ring is regarded as a vortex state. We found that the vorticity shows a nonmonotonic and intriguing dependence on the population imbalance. Second, the FF state changes to the intermediate state through the second order phase transition at $T=T_{\mathrm{c} 2}<T_{\mathrm{c}}$. As decreasing the temperature below $T_{\mathrm{c} 2}$, the crossover occurs from the intermediate state to the LO state. We point out that the FF, LO, and intermediate phases are distinguished by the flux of mass and local polarization. Third, we showed a sequence of quantum phase transitions with increasing the rotation. The FFLO state with an integer vortex changes to the half quantum vortex state. Then, the flux of mass is half quantized (Fig. 3). The half quantum vortex state arises from the multi-component order parameters of the FFLO state, and therefore the half quantized flux would be a clear evidence for the FFLO superfluid state. The spatial profile of the order parameter in the half quantum vortex state is described by the product $\Delta_{\mathrm{FF}} \times \Delta_{\mathrm{LO}}$ where $\Delta_{\mathrm{FF}}$ and $\Delta_{\mathrm{LO}}$ are the order parameters of FF and LO states, respectively.

These results are obtained on the basis of the BdG equation. Since the BdG equation is a mean field approximation it breaks down in the purely one-dimensional system. It is well known that the long-range order of superfluidity/superconductivity is fragile against the fluctuation in one dimension. We adopt the one-dimensional model for simplicity, however our interests are focused on the quasi-one-dimensional superfluid which is realized in the toroidal trap 25, 33, 34]. It has been shown that a weak three-dimensional coupling gives rise to the long range order [43-45]. The BdG equation is qualitatively appropriate for a weak attractive interaction in this case. The correlation effect beyond the mean field approximation plays important roles for a strong attractive interaction. Therefore, our study basically focuses on the weak coupling BCS region. However, it has been shown that the FFLO superfluid state is robust against the strong attractive interaction in the unitary regime [25, 48]. This indicates that the novel FFLO phases studied in this paper are robust in the BCS-BEC crossover region.
We note that a fingerprint of the FFLO phases appears in the purely one-dimensional system [6], although the long range order is suppressed there. For instance, the free energy shows a characteristic periodicity with respect to the phase $\Phi$ as shown in Ref. [53]. We expect that the free energy of the imbalanced gases shows an oscillation with respect to the phase $\Phi$, and the period is a half of the BCS state. This oscillation is a fingerprint of the half quantum vortex state in the FFLO state. It will be interesting to see this periodicity with use of the accurate numerical and/or analytical methods for one-dimensional models.

Three comments are given on the related theoretical works. First, the same model as in Eq. (11) was solved in the recent paper 32] on the basis of the BdG equations. However, they missed most of the novel FFLO phases induced by the rotation, except for the vortex state in the small imbalance region. We showed $T-h$ and $T-P$ phase diagrams in which the FF state with giant vortex, half quantum vortex state, and intermediate state are stabilized. These phases were not shown in Ref. [32].

Second, a superfluid state similar to the A-FFLO state was investigated in the optical lattice by Chen et al. [54]. The response to the rotation is quite different from our case, because the rotation symmetry is broken by the optical lattice potential. For example, the FF state and the intermediate state are suppressed because the degeneracy of the FF states is lifted. In other words, the FFLO state in the optical lattice does not have an internal degree of freedom in contrast to the continuum gases. It is interesting to study the effect of broken rotation symmetry by tuning the aspect ratio [34]. Then, other FFLO states may be stabilized by the rotation.

Third, the rotating FFLO phases studied in this paper are regarded as an analog of the superconducting states in the non-centrosymmetric systems $[55]$. The degeneracy of the FF phases is lifted in the latter case by the anti-symmetric spin-orbit coupling arising from the broken inversion symmetry. The helical superconducting state is an analog of the FF state in our case, while the stripe phase corresponds to the intermediate state. However, these non-centrosymmetric superconducting phases cannot be realized because the orbital de-pairing effect arising from the magnetic field affects the superconducting state. In contrast, cold Fermi gases are ideal system for the study of FFLO phases thanks to their high controllability.

A ring trap has been realized for Bose gases [33, 34], and produces many intriguing topics. We expect that the realization of Fermi superfluid on the ring trap will open a new research field of cold atom gases, as the development of sophisticated trap potentials triggered novel ideas. This paper shows that the rotating FFLO superfluid phases in the imbalanced Fermi gases will be one of the goals. We hope that active investigations will elucidate such novel phases. 


\section{ACKNOWLEDGMENTS}

The authors are grateful to G. Marmorini, S. Tsuchiya, and Y. Ono for fruitful discussions. T. Y. thanks Y. Yamakawa for variable comment on the numerical calculations. This work was supported by a Grant-in-Aid for Scientific Research on Innovative Areas "Heavy Electrons" (No. 21102506) from MEXT, Japan. It was also supported by a Grant-in-Aid for Young Scientists (B) (No. 20740187) from JSPS. Numerical computation in this work was partly carried out at the Yukawa Institute Computer Facility.

\section{Appendix A: Derivation of the Hamiltonian}

In Appendix A, we derive the lattice Hamiltonian in Eq. (1) from the continuum model. The Hamiltonian of continuum one-dimensional gases is described in the rotating coordinate as

$$
\begin{aligned}
H= & \sum_{\sigma} \int d x \hat{\psi}_{\sigma}^{\dagger}(x)\left(-\frac{1}{2 M} \frac{\partial^{2}}{\partial x^{2}}-\Omega \hat{L}_{z}\right) \hat{\psi}_{\sigma}(x) \\
& -|U| \int d x \hat{\psi}_{\uparrow}^{\dagger}(x) \hat{\psi}_{\downarrow}^{\dagger}(x) \hat{\psi}_{\downarrow}(x) \hat{\psi}_{\uparrow}(x), \\
= & H_{0}-|U| \int d x \hat{\psi}_{\uparrow}^{\dagger}(x) \hat{\psi}_{\downarrow}^{\dagger}(x) \hat{\psi}_{\downarrow}(x) \hat{\psi}_{\uparrow}(x),
\end{aligned}
$$

where $\hat{\psi}_{\sigma}^{\dagger}(x)$ and $\hat{\psi}_{\sigma}(x)$ are the fermionic creation and annihilation operators, respectively, and $H_{0}$ is the singleparticle Hamiltonian.

The coordinate along the ring $x$ is given in term of the angle $\theta$ and the radius of ring $R$. Then, the $z$ component of the angular momentum $\hat{L}_{\mathrm{z}}$ is described as $\hat{L}_{z}=-i \frac{\partial}{\partial \theta}=-i R \frac{\partial}{\partial x}$.

With use of the Fourier transformation, the singleparticle Hamiltonian $H_{0}$ is obtained as

$$
H_{0}=\sum_{k, \sigma}\left(\frac{k^{2}}{2 M}-R \Omega k\right) \hat{c}_{k \sigma}^{\dagger} \hat{c}_{k \sigma} .
$$

Thus, the single particle energy is obtained as $\varepsilon(k)=$ $\frac{k^{2}}{2 M}-R \Omega k$. It is understood that the Fermi momentum is shifted to the positive direction of $k$ for $\Omega>0$. On the other hand, the single particle energy in the lattice Hamiltonian (Eq. (1)) is obtained as

$$
\varepsilon(k)=-2 t \cos (k-\Phi) .
$$

This is approximated in the continuum limit $k, \Phi \ll 1$ as

$$
\varepsilon(k) \simeq t\left(\Phi^{2}-2\right)-2 t \Phi k+t k^{2} .
$$

Hence, we obtain the parameters of the lattice Hamiltonian Eq. (1) by which the continuum gases are approximately modeled,

$$
t \leftrightarrow \frac{1}{2 M}, 2 t \Phi \leftrightarrow R \Omega .
$$

\section{Appendix B: Periodicity with respect to $\Phi$}

With use of the gauge transformation,

$$
\hat{c}_{j \sigma} \rightarrow \mathrm{e}^{-i \Phi j} \hat{c}_{j \sigma}
$$

the hopping term is transformed as

$$
-t \hat{c}_{j+1 \sigma}^{\dagger} \hat{c}_{j \sigma} \rightarrow-t \mathrm{e}^{i \Phi} \hat{c}_{j+1 \sigma}^{\dagger} \hat{c}_{j \sigma}
$$

Since we adopt the periodic boundary condition, the phase $\Phi$ should be

$$
\Phi_{n}=\frac{2 \pi}{N_{\mathrm{L}}} n
$$

where $n$ is an integer. This gauge transformation changes the Hamiltonian for the gas at rest to that for the rotating gas. This means that the physical quantities are periodic in $\Phi$ with the period $2 \pi / N_{\mathrm{L}}=\Phi_{0}$. Hence we show the results for $0 \leq \Phi / \Phi_{0}<1$ in Figs. 2 and 7 .
[1] G. B. Partridge, W. Li, R. I. Kamar, Y.-a. Liao, and R. G. Hulet, Science 311, 503 (2006).

[2] M. W. Zwierlein, A. Schirotzek, C. H. Schunck, and W. Ketterle, Science 311, 492 (2006).

[3] S. Giorgini, L. P. Pitaevskii, and S. Stringari, Rev. Mod. Phys. 80, 1215 (2008)

[4] P. Fulde and R. A. Ferrell, Phys. Rev. 135, A550 (1964)

[5] A. I. Larkin and Y. N. Ovchinnikov, Sov. Phys. JETP 20, 762 (1965).

[6] Y.-a. Liao, A. S. C. Rittner, T. Paprotta, W. Li, G. B. Partridge, R. G. Hulet, S. K. Baur, and E. J. Mueller, Nature (London) 467, 567 (2010).

[7] H. A. Radovan, N. A. Fortune, T. P. Murphy, S. T. Hannahs, E. C. Palm, S. W. Tozer, and D. Hall, Nature (London) 425, 51 (2003).
[8] A. Bianchi, R. Movshovich, C. Capan, P. G. Pagliuso, and J. L. Sarrao, Phys. Rev. Lett. 91, 187004 (2003).

[9] Y. Matsuda and H. Shimahara, J. Phys. Soc. Jpn. 76, 051005 (2007).

[10] M. Kenzelmann, T. Strässle, C. Niedermayer, M. Sigrist, B. Padmanabhan, M. Zolliker, A. D. Bianchi, R. Movshovich, E. D. Bauer, J. L. Sarrao, and J. D. Thompson, Science 321, 1652 (2008).

[11] R. Ikeda, Phys. Rev. B 76, 134504 (2007)

[12] R. Ikeda, Phys. Rev. B 76, 054517 (2007)

[13] D. F. Agterberg, M. Sigrist, and H. Tsunetsugu, Phys. Rev. Lett. 102, 207004 (2009)

[14] A. Aperis, G. Varelogiannis, and P. B. Littlewood, Phys. Rev. Lett. 104, 216403 (2010)

[15] Y. Yanase and M. Sigrist, J. Phys. Soc. Jpn. 78, 114715 (2009). 
[16] R. Casalbuoni and Rev. Mod. Phys. 76, 263 (2004)

[17] D. E. Sheehy and L. Radzihovsky, Phys. Rev. Lett. 96, 060401 (2006)

[18] N. Yoshida and S.-K. Yip, Phys. Rev. A 75, 063601 (2007)

[19] P. Castorina, M. Grasso, M. Oertel, M. Urban, and D. Zappalà, Phys. Rev. A 72, 025601 (2005).

[20] J. Kinnunen, L. M. Jensen, and P. Törmä, Phys. Rev. Lett. 96, 110403 (2006).

[21] T. Mizushima, M. Ichioka, and K. Machida, J. Phys. Soc. Jpn. 76, 104006 (2007).

[22] K. Machida, T. Mizushima, and M. Ichioka, Phys. Rev. Lett. 97, 120407 (2006).

[23] X.-J. Liu, H. Hu, and P. D. Drummond, Phys. Rev. A 75, 023614 (2007)

[24] M. Tezuka, Y. Yanase, and M. Ueda, e-print (2008), arXiv:0811.1650.

[25] Y. Yanase, Phys. Rev. B 80, 220510 (2009).

[26] T. Kashimura, S. Tsuchiya, and Y. Ohashi, Phys. Rev. A 84, 013609 (2011)

[27] P. Nikolić, Phys. Rev. A 81, 023601 (2010)

[28] M. L. Kulić, A. Sedrakian, and D. H. Rischke, Phys. Rev. A 80, 043610 (2009)

[29] A. V. Samokhvalov, A. S. Mel'nikov, and A. I. Buzdin, Phys. Rev. B 82, 174514 (2010)

[30] A. V. Samokhvalov, A. S. Mel'nikov, and A. I. Buzdin, Phys. Rev. B 76, 184519 (2007)

[31] A. A. Zyuzin and A. Y. Zyuzin, Phys. Rev. B 79, 174514 (2009)

[32] H. T. Quan and J.-X. Zhu, Phys. Rev. B 81, 014518 (2010)

[33] C. Ryu, M. F. Andersen, P. Cladé, V. Natarajan, K. Helmerson, and W. D. Phillips, Phys. Rev. Lett. 99, 260401 (2007).

[34] B. E. Sherlock, M. Gildemeister, E. Owen, E. Nugent, and C. J. Foot, Phys. Rev. A 83, 043408 (2011).

[35] K. Yang, Phys. Rev. B 63, 140511 (2001)
[36] G. Orso, Phys. Rev. Lett. 98, 070402 (2007).

[37] M. Tezuka and M. Ueda, Phys. Rev. Lett. 100, 110403 (2008)

[38] H. Hu, X.-J. Liu, and P. D. Drummond, Phys. Rev. Lett. 98, 070403 (2007).

[39] X.-J. Liu, H. Hu, and P. D. Drummond, Phys. Rev. A 76, 043605 (2007).

[40] A. E. Feiguin and F. Heidrich-Meisner, Phys. Rev. B 76, 220508 (2007).

[41] A. E. Feiguin and F. Heidrich-Meisner, Phys. Rev. Lett. 102, 076403 (2009)

[42] M. Rizzi, M. Polini, M. A. Cazalilla, M. R. Bakhtiari, M. P. Tosi, and R. Fazio, Phys. Rev. B 77, 245105 (2008).

[43] M. M. Parish, S. K. Baur, E. J. Mueller, and D. A. Huse, Phys. Rev. Lett. 99, 250403 (2007).

[44] E. Zhao and W. V. Liu, Phys. Rev. A 78, 063605 (2008)

[45] R. M. Lutchyn, M. Dzero, and V. M. Yakovenko, Phys. Rev. A 84, 033609 (2011).

[46] Q. Chen, J. Stajic, S. Tan, and K. Levin, Phys. Rep. 412, 1 (2005)

[47] F. Heidrich-Meisner, A. E. Feiguin, U. Schollwöck, and W. Zwerger, Phys. Rev. A 81, 023629 (2010).

[48] A. Bulgac and M. M. Forbes, Phys. Rev. Lett. 101, 215301 (2008)

[49] K. Machida and H. Nakanishi, Phys. Rev. B 30, 122 (1984)

[50] R. Yoshii, S. Tsuchiya, G. Marmorini, and M. Nitta, Phys. Rev. B 84, 024503 (2011)

[51] A. B. Vorontsov and M. J. Graf, Phys. Rev. B 74, 172504 (2006)

[52] D. A. Ivanov, Phys. Rev. Lett. 86, 268 (2001)

[53] A. Sudbø, C. M. Varma, T. Giamarchi, E. B. Stechel, and R. T. Scalettar, Phys. Rev. Lett. 70, 978 (1993)

[54] Y. Chen, Z. D. Wang, F. C. Zhang, and C. S. Ting, Phys. Rev. B 79, 054512 (2009).

[55] D. F. Agterberg and R. P. Kaur, Phys. Rev. B 75, 064511 (2007) 